\title{
The miR 302-367 cluster drastically affects self-renewal and infiltration properties of glioma-initiating cells through CXCR4 repression and consequent disruption of the SHH-GLI-NANOG network
}

\author{
M Fareh ${ }^{1}$, L Turchi ${ }^{1}$, V Virolle ${ }^{1}$, D Debruyne ${ }^{1}$, F Almairac ${ }^{1,2}$, S de-la-Forest Divonne ${ }^{1}$, P Paquis ${ }^{2}, O$ Preynat-Seauve ${ }^{3}$, K-H Krause ${ }^{3}$, \\ H Chneiweiss ${ }^{4}$ and T Virolle ${ }^{*, 1}$
}

Glioblastoma multiforme (GBM) is the most common form of primary brain tumor in adults, often characterized by poor survival. Glioma-initiating cells (GiCs) are defined by their extensive self-renewal, differentiation, and tumor initiation properties. GiCs are known to be involved in tumor growth and recurrence, and in resistance to conventional treatments. One strategy to efficiently target GiCs in GBM consists in suppressing their stemness and consequently their tumorigenic properties. In this study, we show that the miR-302-367 cluster is strongly induced during serum-mediated stemness suppression. Stable miR-302-367 cluster expression is sufficient to suppress the stemness signature, self-renewal, and cell infiltration within a host brain tissue, through inhibition of the CXCR4 pathway. Furthermore, inhibition of CXCR4 leads to the disruption of the sonic hedgehog (SHH)GLI-NANOG network, which is involved in self-renewal and expression of the embryonic stem cell-like signature. In conclusion, we demonstrated that the miR-302-367 cluster is able to efficiently trigger a cascade of inhibitory events leading to the disruption of GiCs stem-like and tumorigenic properties.

Cell Death and Differentiation (2012) 19, 232-244; doi:10.1038/cdd.2011.89; published online 1 July 2011

Glioblastoma multiforme (GBM) is the most common form of primary brain tumor in adults, with a median survival rate of 14 months. ${ }^{1}$ GBM is a highly vascularized and infiltrating tumor that can develop lesions at sites distant from the primary tumor. ${ }^{2,3}$ Treatments for GBM consist of surgical resection combined with radiotherapy plus temozolomide, which has been found to significantly increase survival, ${ }^{1}$ especially in patients exhibiting epigenetic silencing of the 0-6 methylguanine-DNA methyltransferase (MGMT) gene. ${ }^{4}$ However, this approach remains largely ineffective and leads to tumor recurrences. Failure to cure GBM has been attributed to the extensive resistance of glioma-initiating cells (GICs) to conventional cytotoxic therapies. The 'GICs hypothesis' proposes that a minority of highly tumorigenic and selfrenewing cells in the tumor bulk, are responsible for tumor initiation and behavior. ${ }^{5}$ The concept of tumor stem cells, originally proposed for myeloid leukemia, ${ }^{6}$ was subsequently shown to be valid for solid tumors as well. ${ }^{7-9}$ A recent report showed that GICs become vulnerable to therapies, and more importantly lose their tumorigenic ability, when they are forced to mature into a more differentiated state. ${ }^{10}$ Therefore, the identification of ways to efficiently affect GICs properties may lead to the development of new therapeutic approaches for the treatment of malignant glioma. In this context, it is crucial to investigate the mechanisms involved in GICs plasticity, which is likely the result of a tight epigenetic control of gene expression. Micro-RNAs modulate gene expression levels by promoting either inhibition of translation or mRNAs degradation. Their involvement in self-renewal and stem cells plasticity is sustained by recent studies performed in human embryonic stem cells ${ }^{11,12}$

In the present study, we show that the miR 302-367 cluster is rapidly and strongly induced in GiCs undergoing serummediated suppression of stemness. This miR cluster is undetectable in self-renewing GiCs; however, when stably and constitutively expressed, it is able to suppress the stemness gene signature, self-renewal, and cell infiltration through the inhibition of the CXCR4 pathway. In addition, we show that blockade of CXCR4 signaling leads to the disruption of the sonic hedgehog (SHH)-GLI-NANOG network, which is known to be responsible for self-renewal and expression of the ES-like genes signature in glioma cancer stem cells. ${ }^{13}$

\section{Results}

The miR-302-367 cluster contributes to serum-mediated loss of stemness. To identify miRNAs capable of

${ }^{1}$ INSERM U898/UNSA, Cellules Souches, Développement et Cancer, faculté de médecine, av valombrose, Nice, France; ${ }^{2}$ Service de Neurchirurgie, hôpital Pasteur, $\mathrm{CHU}$ de Nice, Nice, France; ${ }^{3}$ Department of Pathology and Immunology, Faculty of Medicine, University of Geneva and Department of Genetic and Laboratory Medicine, Geneva Hospital, Geneva, Switzerland and ${ }^{4}$ U894 Inserm/Unversité Paris-Descartes, Plasticité Gliale, Centre de Psychiatrie et Neurosciences, Paris, France ${ }^{*}$ Corresponding author: T Virolle, INSERM U898/UNSA, Cellules Souches, Développement et Cancer, faculté de médecine, av valombrose, Nice 06107, France. Tel: + 049337 7620; Fax: + 049337 7051; E-mail: virolle@ unice.fr Keywords: miRNA; self-renewal; cancer stem cells; cxcr4; infiltration

Abbreviations: GBM, Glioblastoma multiforme; MGMT, 0-6 methylguanine-DNA methyltransferase; GICS, glioma-initiating cells; miRNA, micro ribonucleic acid; SHH, sonic hedgehog; EGF, epidermal growth factor; bFGF, basic fibroblast growth factor; GFAP, glial fibrillary acidic protein; IPS, inducible pluripotent stem cells Received 14.12.10; revised 20.4.11; accepted 17.5.11; Edited by R De Maria; published online 01.7.11 
suppressing GiCs stemness properties, we used two previously characterized GiCs primary lines, ${ }^{14}$ TG1 and TG6, and took advantage of the ability of serum to induce stem cells differentiation. ${ }^{14}$ Self-renewing TG cells were forced into a more differentiated non-stem-like state, by substituting the growth factors EGF and bFGF with $0.5 \%$ serum. After 4 days, the glial proteins GFAP and Olig2 were upregulated (Figures $1 \mathrm{a}$ and $\mathrm{b}$ ) while the cells exhibited low levels of the stemness markers Oct-4, Nanog, (Supplementary Figure 1A), and of SHH clearly established as a major player in GiCs self-renewal ${ }^{15}$ (Figures 1a and b). Consistent with $\mathrm{SHH}$ repression, serum treatment drastically reduced clonal proliferation (Figure 1b). These results confirmed that serum-treated TG cells are an ideal system to identify molecules involved in loss of stem-like properties. Hence, we compared the human miRNA signature of selfrenewing TG1 and TG6 cells with that of 4-day serumdifferentiated cells. Three independent experiments revealed about 60 miRNAs modulated at this time point. As our goal was to identify miRNAs able to promote loss of stemness, we took into account only the most upregulated miRNAs and focused on the miR-302-367 cluster (Figure 1c) whose members (miR-302a, b, c, d, and miR-367) were exclusively expressed in serum-differentiated cells (Supplementary Figure 1B). A time course analysis revealed the induction of this cluster at day 1, a maximal expression at day 3 (Figure 1d; Supplementary Figure 1C), which was maintained up to 15 days following serum addition. Similar results were obtained using two additional GiCs primary lines, GB1 and \#1056 (Supplementary Figure 1D). In order to assess miR-302-367 cluster contribution in serum-mediated suppression of stemness, we transiently transfected TG1 cells with anti-miR-302a, anti-miR302b or anti-miR-367 (Figure 1e), or with a pool of these three anti-miRs (Figure 1f). After $24 \mathrm{~h}$, the cells were treated with serum. Quantitative real-time PCR (QPCR) analysis revealed that the aforementioned anti-miRs were able to reduce the expression of each $\mathrm{miR}$ by $50 \%$, whether they were used individually or as a pool (Supplementary Figures 2A and B). Although individual anti-miR did not affect the differentiation process (Figure 1e), the pool was able to significantly alter the expression of GFAP, Olig2, and SHH (Figure 1f), suggesting compensatory mechanisms. These results demonstrate a functional contribution of the miR-302-367 cluster in serum-mediated suppression of stemness.

The miR-302-367 cluster promotes suppression of stemness and contributes to GiCs glial commitment. To assess the capacity of the miR-302-367 cluster to suppress stemness in the absence of serum, we cloned the genomic region containing the whole miR-302-367 cluster into a lentiviral vector. We used this construct to generate the TG1 Cluster 302 and TG6 Cluster 302\#1 cell lines, which stably expressed each miR-302-367 cluster member (Figures 2a and b). As control, we used TG1 and TG6 cells expressing a non-relevant small non-coding RNA (TG1 and TG6 Ctrl cells). To assess the miR-302-367 cluster ability to trigger differentiation in the absence of serum, we cultured TG Ctrl and TG Cluster 302 cells in a minimum defined medium deprived of EGF and bFGF (NS34-), for
4 days. This medium is sufficient to maintain a stem-like state while preventing spontaneous differentiation of control cells. In these conditions, TG1 and TG6 Ctrl cells grew as nonadherent neurospheres (Figures $2 a$ and b), while TG1 and TG6 Cluster 302 cells grew adherent and exhibited a nonstem-like differentiated phenotype (Figures $2 a$ and b). QPCR analysis showed an increased expression of GFAP and a reduced expression of $\mathrm{SHH}$ in the TG1 Cluster 302 and TG6 Cluster 302\#1 as compared with the Ctrl cells (Figure 2c). We then studied the expression of stemness markers by immunofluorescence. Although $\mathrm{SHH}$, Oct4, Nanog, and Sox1 were clearly expressed in TG1 Ctrl cells cultured in growing medium (NS34+) (Figure 3a), they were almost undetectable in TG1 Cluster 302 cells cultured under the same conditions (Figure 3a). When TG1 Cluster 302 cells were cultured in NS34-, GFAP was strongly expressed (Figure $3 b$ ) while, unlike cells treated with serum, neuronal markers such as NeuN remained undetectable (Figure $3 b$ ). Similar results were obtained using TG6 Cluster $302 \# 1$ cells (Supplementary Figures $3 \mathrm{~A}, \mathrm{~B}$ and $\mathrm{C}$ ). These results demonstrate that expression of the miR-302-367 cluster is sufficient, in a context favorable for GiCs self-renewal, to promote loss of stemness markers and to contribute, in quiescent conditions, to early tumor glial fate commitment.

The miR-302-367 cluster expression drastically inhibits GiCs self-renewal and infiltration properties. The loss of stemness markers mediated by miR-302-367 cluster strongly suggests an impairment of stem-like properties. We therefore evaluated the clonal efficiency of TG1 Cluster 302 cells compared with TG1 Ctrl cells. We seeded 10 cells/ well in a 96-wells plate and considered clonal efficiency to be $100 \%$ when, after 1 month, 10 neurospheres were counted in a well. Although $80 \%$ of single TG1 Ctrl cells were able to form neurospheres, less than $10 \%$ of TG1 Cluster 302 cells were able to do so (Figure 4a). Therefore, miR-302-367 cluster inhibited clonal proliferation of more than $80 \%$ (Figure 4a). Similar results were obtained using TG6 Cluster 302\#1 and TG6 Ctrl cells (Supplementary Figure 3D). Importantly, the size of the neo-formed neurospheres in TG1 Cluster 302 cells was much smaller than the one observed in TG1 Ctrl cells (Figure 4a), suggesting a reduction of the proliferation rate. To compare the cell-cycle characteristics of TG1 Cluster 302 cells with those of TG1 Ctrl cells, we analyzed their cell-cycle profiles by FACS (Figure 4b). When compared with TG1 Ctrl cells, TG1 Cluster 302 cells exhibited an accumulation of cells in the G1 phase (59.49 versus $51.76 \%$ ). The lower proliferation rate of TG1 Cluster 302 cells was confirmed using proliferation assays (Figure 4c). To further assess the impact of such a growth impairment on the development of an in vitro 3D tumor-like cellular mass, we seeded TG1 Ctrl and TG1 Cluster 302 cells, previously labeled with the red fluorescent protein, on separate nylon filters in a minimum medium and under air-liquid interface conditions. After 1 month, a cellular mass can be visualized. Our results showed that TG1 Ctrl cells developed into a thick and compact tissue, whereas TG1 Cluster 302 cells failed to do so and appeared as a thin monolayer (Figure 4d). To assess TG1 or TG6 Cluster 302 cells ability to infiltrate and 

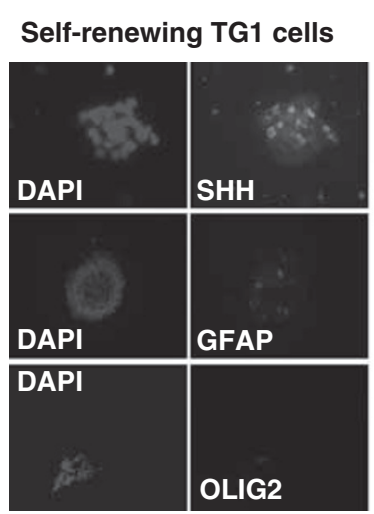

Serum-differentiated

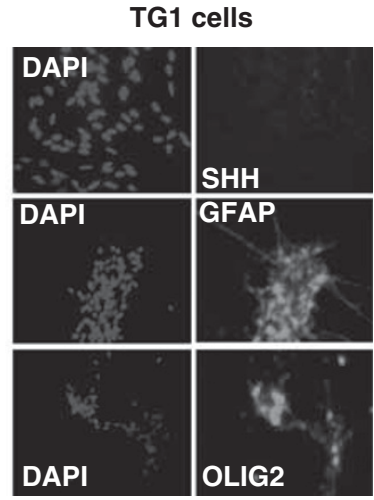

b

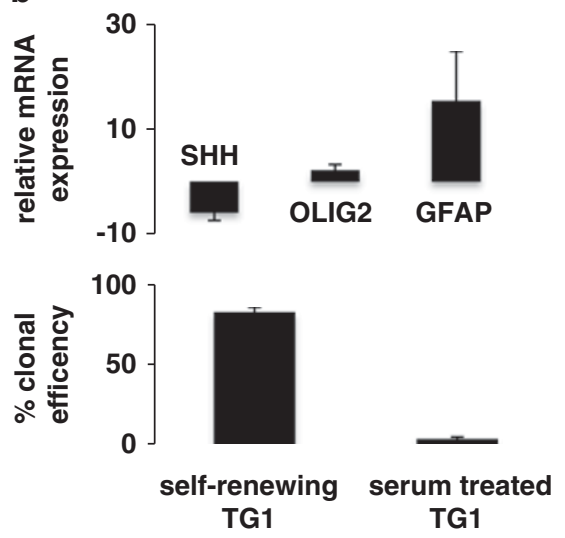

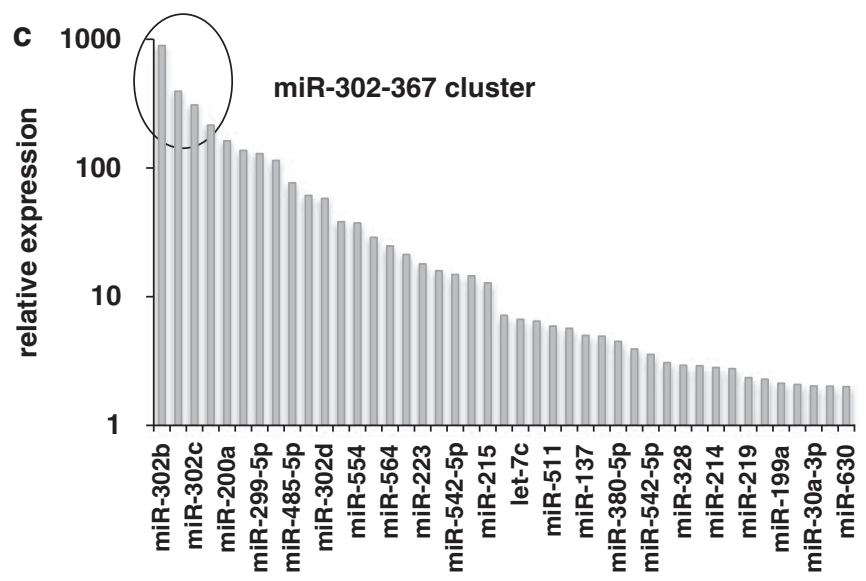
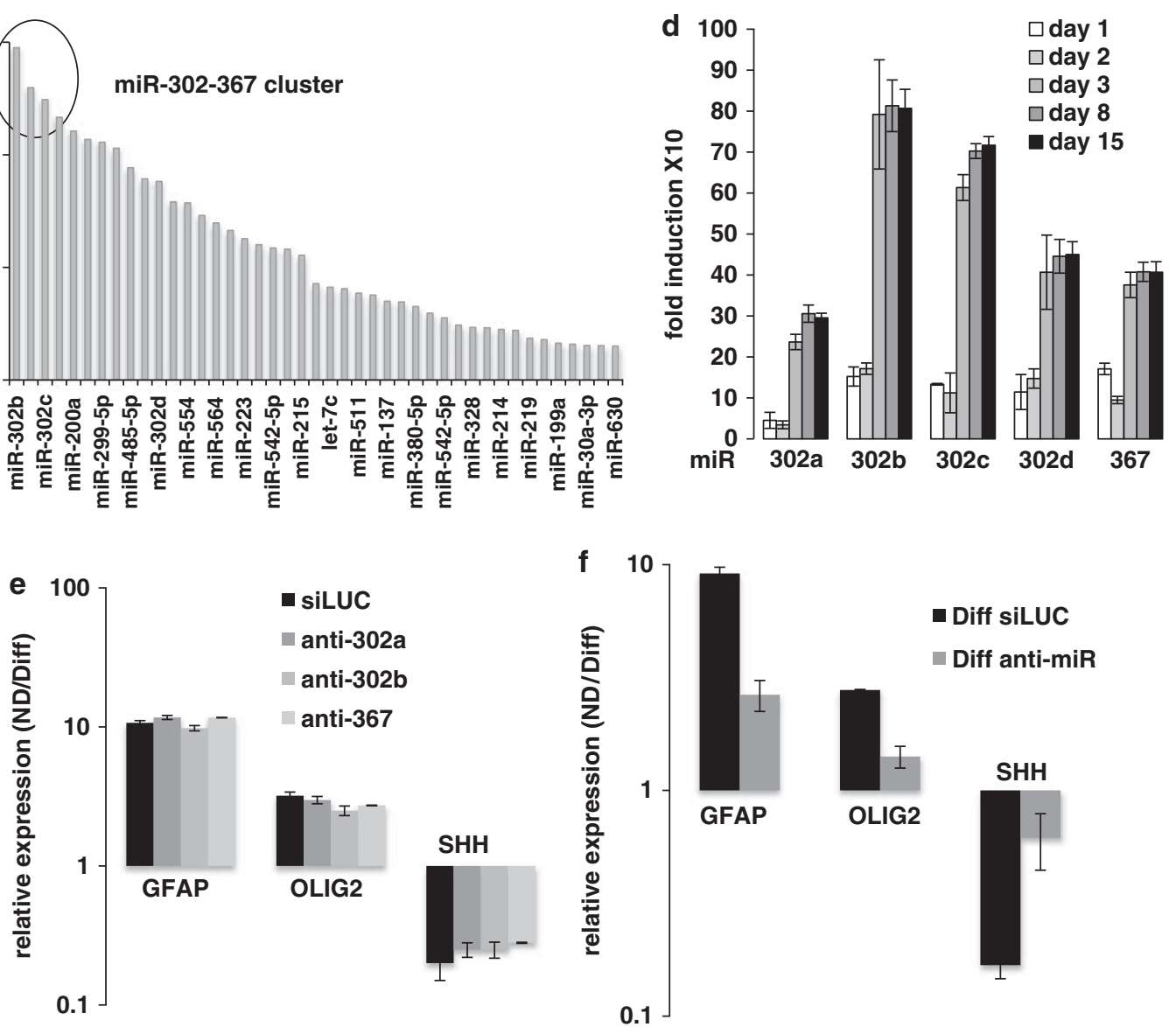

Diff siLUC

Diff anti-miR
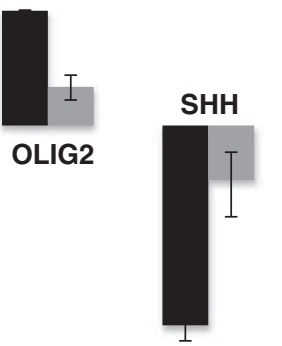

Figure 1 The miR-302-367 cluster contributes to serum-mediated suppression of GiCs stemness. (a) Immunofluorescence and (b, upper panel) QPCR were used to detect SHH and the glial markers GFAP and OLIG2 expression in self-renewing or serum-differentiated TG1 cells. The lower panel of (b) represents the clonal efficiency of self-renewing and serum-treated TG1 cells. (c) Measurement of miRNAs overexpressed after 4 days of serum-mediated differentiation, by Taqman low-density arrayTLDA. The circle highlights the miR-302-367 cluster. (d) QPCR analysis of the miR-302-367 cluster at different time points (1, 2, 3, 8, and 15 days) of serum-mediated differentiation. Self-renewing TG1 cells were cultured either in their defined medium or in $0.5 \%$ serum to induce differentiation. At each time point, total RNA was extracted and expression of miR-302a, b, c, d, and 367 was determined using Taqman probe as described in the Materials and Methods section. (e) Anti-miR-302a, anti-miR-302b, anti-miR-367, or (f) a pool of the three anti-miRs were used to transiently transfect TG1 cells to determine the importance of individual or simultaneous functional inhibition of these specific $\mathrm{miR}$ in serum-mediated differentiation (Diff). After 4 days of transfection, SHH, GFAP, and OLIG2 expression were assessed by QPCR. siLUC was used as control

proliferate within a cerebral tissue, we used ex-vivo organotypic cultures of thick mouse brain slices (MBSs) (Figure 4e). We seeded red fluorescent TG Ctrl or TG Cluster 302 cells on the surface of MBSs and allowed them to grow for 3 weeks. Our results showed a strong ability of Ctrl cells to infiltrate and proliferate within the cerebral tissue, initiating the development of a tumor-like mass in the vicinity of individual migrating cells (Figure $4 \mathrm{e}$ and 
a

Stable expression
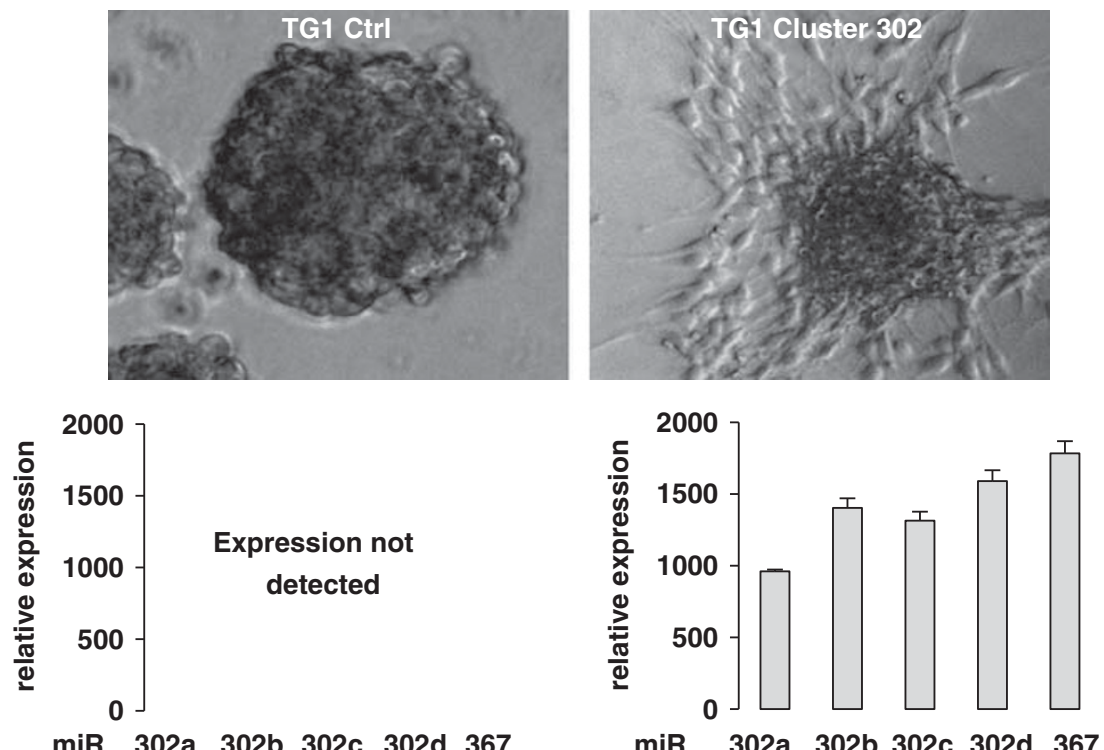

$\operatorname{miR} \quad 302 a \quad 302 b \quad 302 c \quad 302 d 367$

$\operatorname{miR} \quad 302 a \quad 302 b \quad 302 c \quad 302 d \quad 367$

b
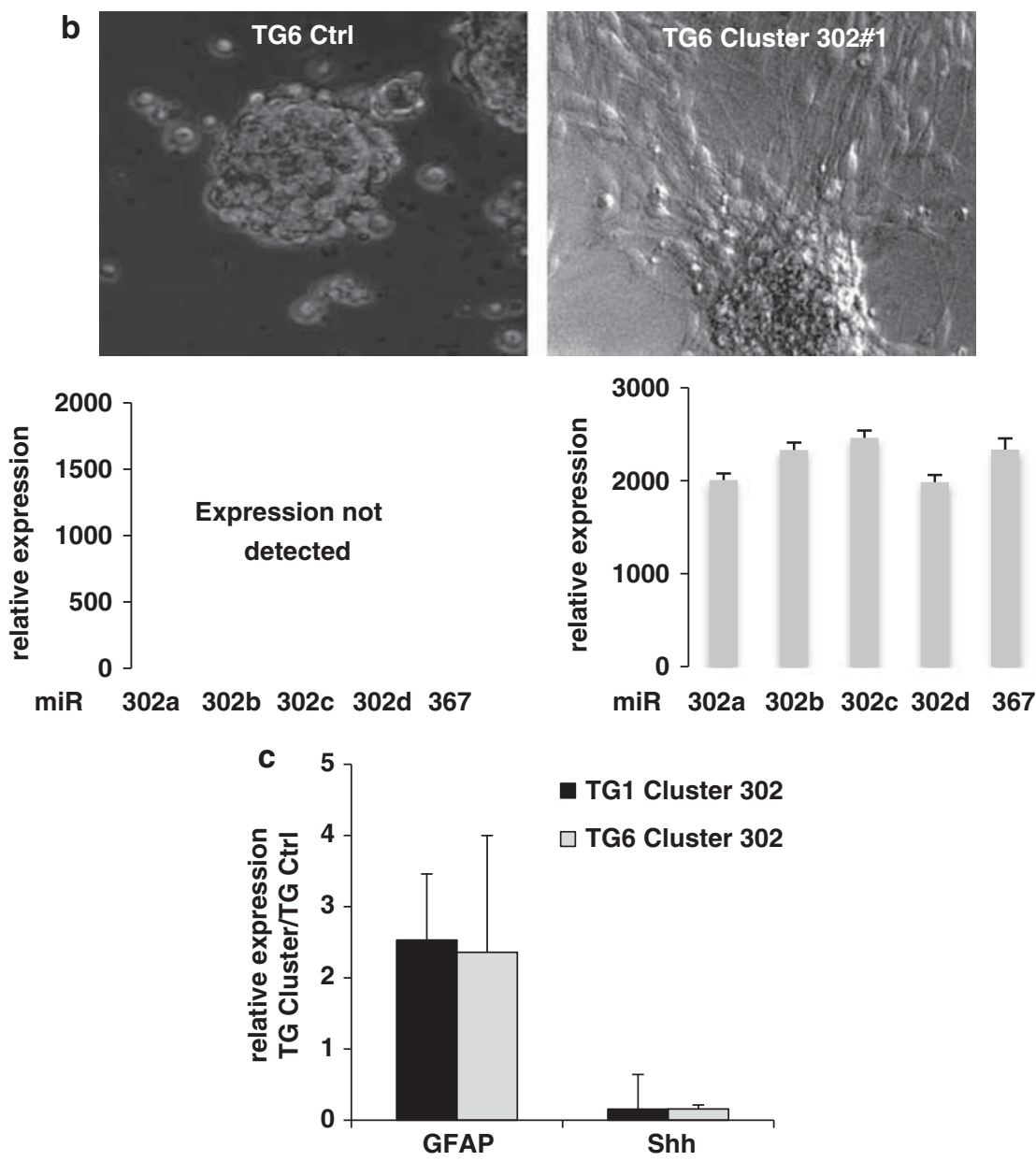

Figure 2 miR-302-367 cluster expression promotes a phenotype similar to that of differentiated cells. Stable primary cell lines TG1 (a) and TG6 (b) expressing the miR-302-367 cluster (TG1 Cluster 302 and TG6 Cluster 302\#1) or a non-relevant small non-coding RNA construct (TG1 Ctrl and TG6 Ctrl) were generated. Relative expression of each miRNA of the miR-302-367 cluster was assessed by QPCR using Taqman probes in TG1 Cluster 302, TG2 Cluster 302\#1, and their respective control cells (lower panels). Upon EGF and bFGF removal, only TG1 Cluster 302 and TG2 Cluster 302\#1 cells became adherent and exhibited a morphology typical of differentiated cell (pictures on the right), while TG1 and TG6 Ctrl cells remained unchanged. (c) Expression of GFAP and SHH, in TG Cluster 302 cells compared with TG Ctrl cells was assessed by QPCR after 4 days of culture in bFGF- and EGF-depleted medium. Error bars are derived from three independent experiments 
a TG1 Ctl in NS34+

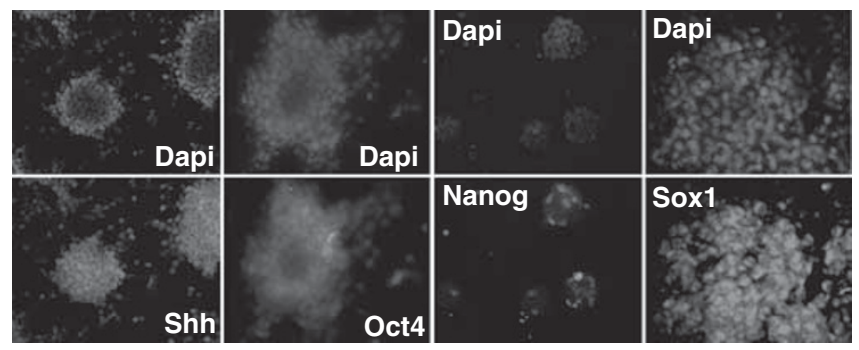

TG1 Cluster 302 in NS34+
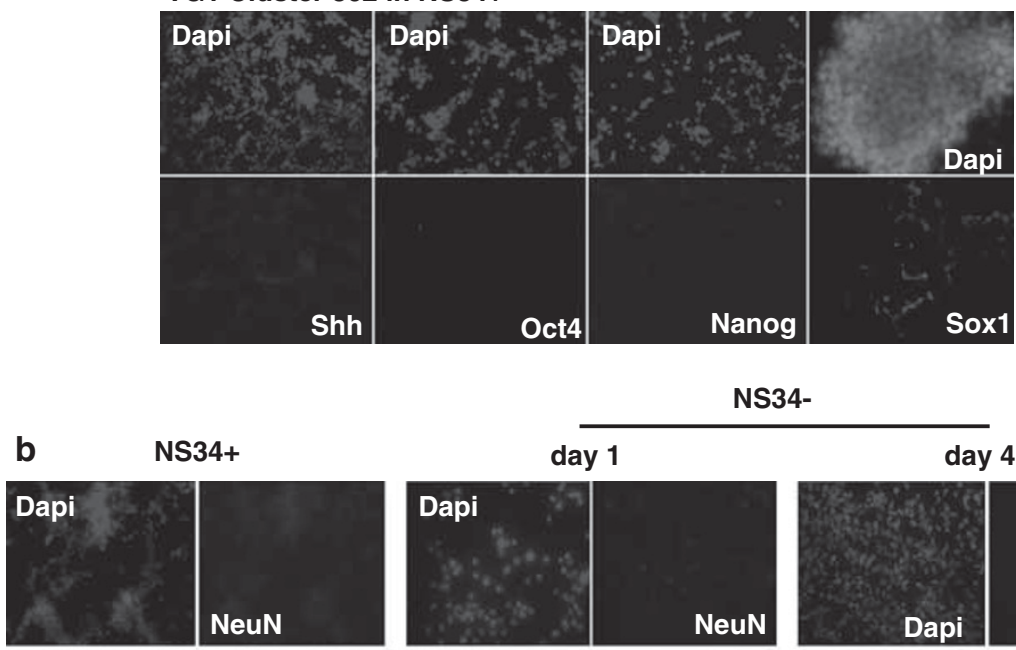

NS34-

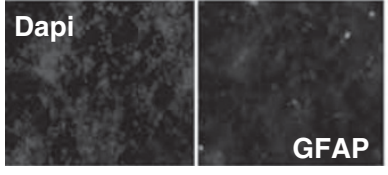

TG1 Cluster 302

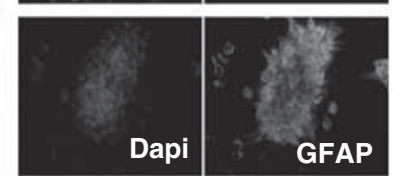

TG1 Cluster 302

Figure 3 Stable miR-302-367 cluster expression induces loss of stemness and upregulation of glial markers. (a) Expression of stemness markers (SHH, Oct4, Nanog, and Sox1) was evaluated by immunofluorescence in TG1 Ctrl (upper panels) or TG1 Cluster 302 cells (lower panels). Cells were cultured in growing medium containing EGF and bFGF (NS34 +), which are required for GiCs proliferation and self-renewal. (b) Expression of glioneuronal markers (GFAP and NeuN) was evaluated by immunofluorescence in TG1 Cluster 302 cells cultured either in NS34 + (left panel) or in medium depleted of EGF and bFGF (NS34-) (day 1, middle panel; day 4 right panel). Nuclei were stained with DAPI

Supplementary Figure 3E). Conversely, TG Cluster 302 cells failed to penetrate the tissue and remained on the surface of the brain slice (Figure $4 \mathrm{e}$ and Supplementary Figure 3E). Under certain circumstances, lack of interaction with the brain tissue even led to loss of the cells (Figure 4e; Supplementary Figure $3 \mathrm{E}$ ). To assess the tumorigenic potential of TG1 Ctrl and TG1 Cluster 302 cells in vivo, we performed intracranial xenografts in NOD/SCID mice using either TG1 Ctrl or TG1 Cluster 302 cells stably expressing a luciferase reporter gene. Before injection, the luciferase activity was assessed by an in vitro luciferase assay and was found to be equivalent in the two cell lines. Time course analysis following the xenograft showed that while TG1 Ctrl cells induced and maintained a tumor for at least 1 month, the TG1 Cluster 302 cells failed to do so (Figures 5a and b). These results demonstrate that miR-302-367 cluster expression is sufficient to inhibit clonal proliferation and infiltration, therefore compromising GiCs tumorigenic properties.
The miR-302-367 cluster expression leads to a drastic downregulation of CXCR4/SDF1. Overexpression of CXCR4, a tumorigenic and invasiveness marker, often characterizes neural cancer stem cells as well as many other cancer cells. ${ }^{16}$ In silico analysis (Miranda, pictar vert) indicated that the CXCR4 $3^{\prime}$ UTR end as well as the $3^{\prime}$ UTR end of its ligand CXCL12/SDF1 might be directly targeted by miR-302a, b, c, and d (Figure 6a). To assess whether this putative regulation occurs in our system, we transiently transfected TG1 cells with the miR-302-367 cluster and compared CXCR4 and SDF1 mRNA levels with those of TG1 cells transfected with a non-relevant construct. Our results indicated a strong miR-302-367 cluster-mediated inhibition of CXCR4 and SDF1 expression (Figure 6b). Accordingly, CXCR4 protein level was significantly reduced in TG1 Cluster 302 cells when compared with TG1 Ctrl cells (Figure 6e). Functional inhibition of at least two cluster members (302a and $302 \mathrm{~b}$ ) was sufficient to partially rescue CXCR4 and SDF1 expression in TG1 Cluster 302 cells 
a

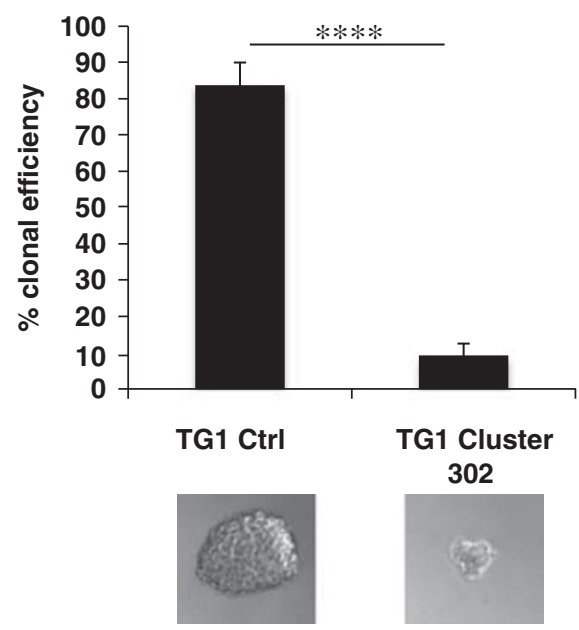

C

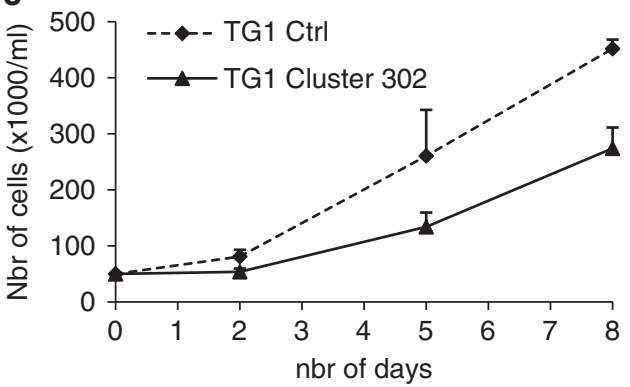

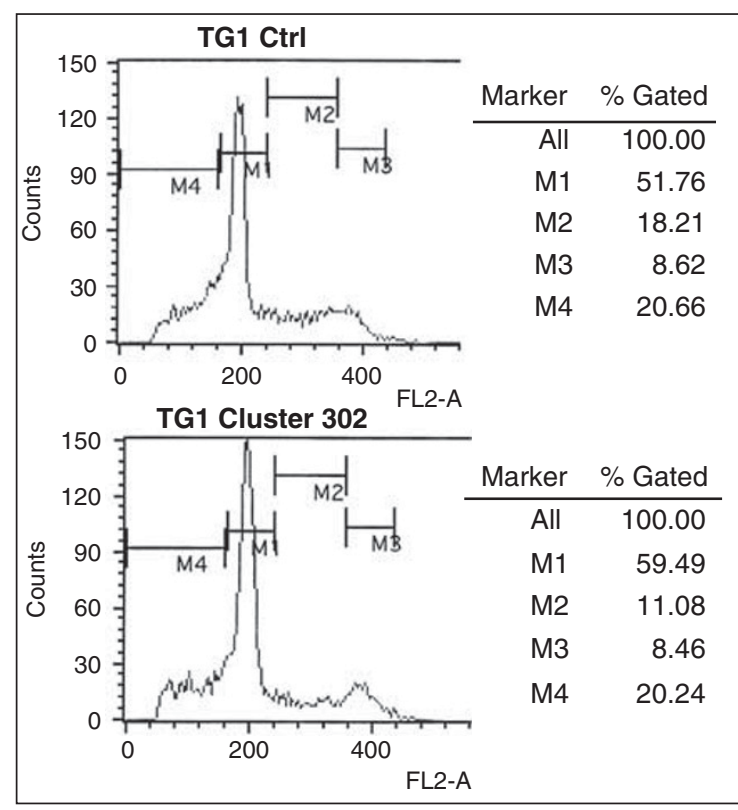

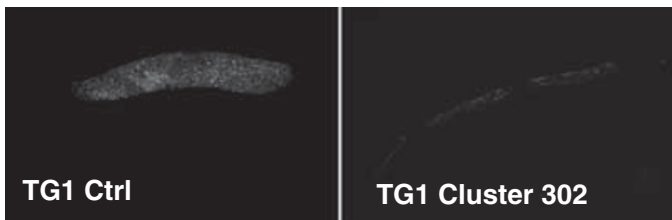

e
TG1 Ctrl
TG1 Cluster 302
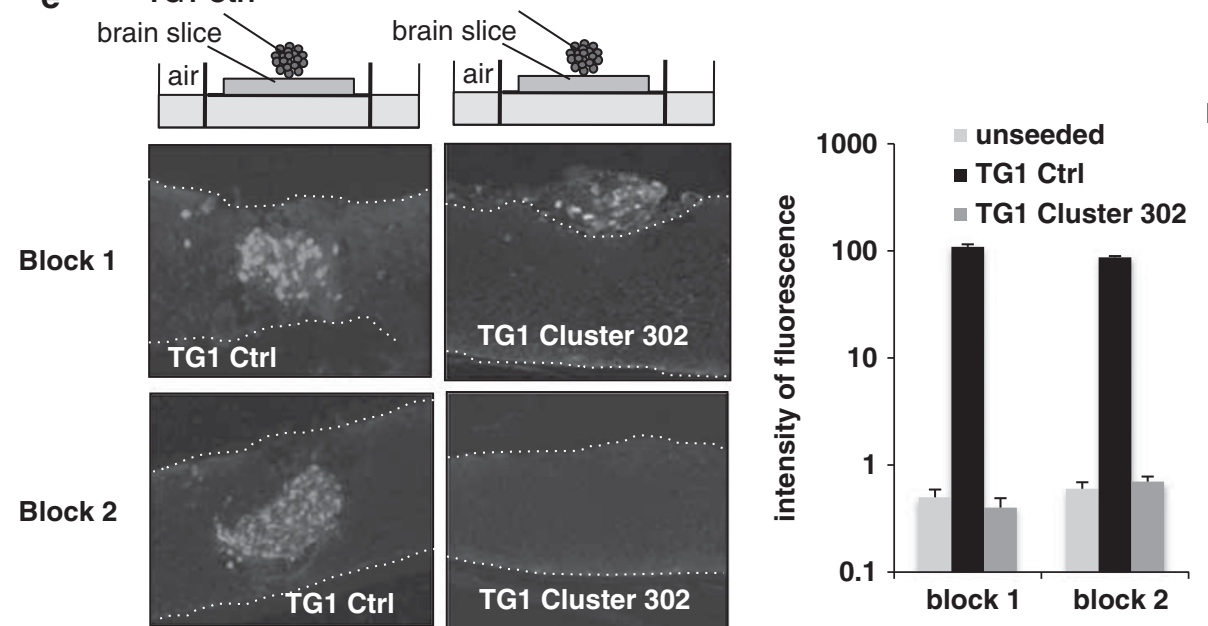

Block 2 (top surface)
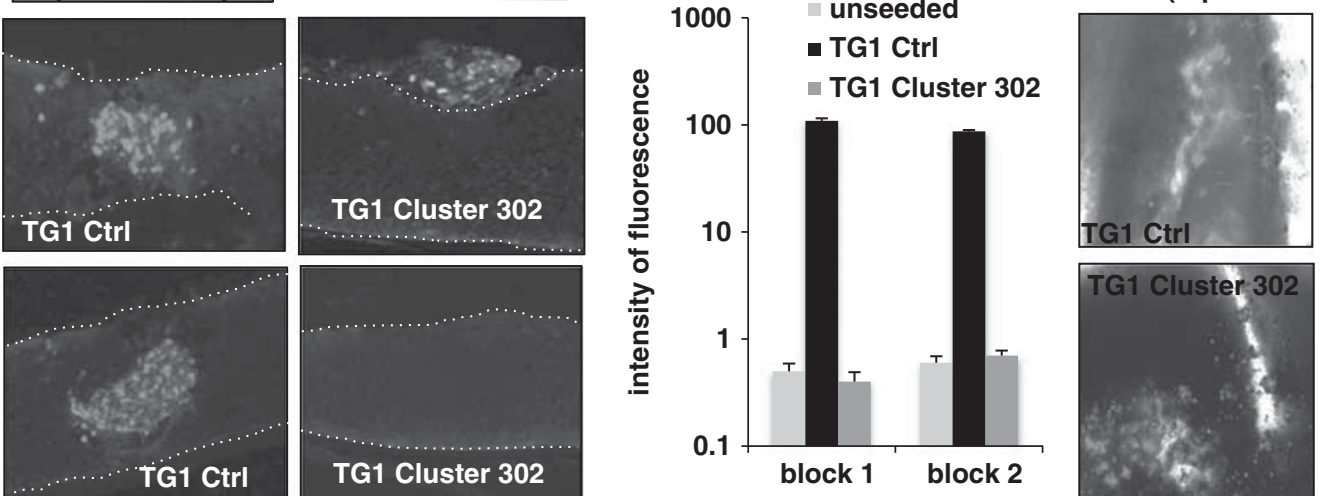

TG1 Cluster 302

Figure 4 The miR-302-367 cluster inhibits clonogenicity and infiltrative properties of TG1 GiCs. (a) TG1 Ctrl and TG1 Cluster 302 cells were seeded at a density of 10 cells/well in a 96-wells plate and allowed to grow. After 1 month, the neurospheres were counted; 10 neurospheres/well corresponded to a clonal efficiency of $100 \%$. Error bars are derived from three independent experiments. Statistical analysis was performed using Student's $t$-test: **** corresponds to a $P$-value of $4.410^{-18}$ and indicates a highly signicant difference between the clonal efficiency of TG1 Ctrl and TG1 Cluster 302 cells. The pictures below the plot show the size difference between the neurospheres formed by TG1 Ctrl cells and those formed by TG1 Cluster 302 cells. (b) The DNA content of TG1 Ctrl and TG1 Cluster 302 cells was labeled with propidium iodide. Cell-cycle profile was assessed by FACS. (c) 80000 TG1 Ctrl (solid line) and TG1 Cluster 302 (dashed line) cells were seeded in a 35-mm diameter culture dish. The cells were then counted at each indicated time point. Error bars are derived from three independent experiments. (d) TG1 Ctrl and TG1 Cluster 302 cells stably expressing the red fluorescent protein were seeded on a nylon filter under airliquid interface conditions as described in the Materials and Methods and allowed to grow. After 1 month, the filter was embedded in paraffin and sliced. The red fluorescent protein showed the growth and development of a tumor-like tissue in vitro only in the case of TG1 Ctrl cells. (e) TG1 Ctrl and TG1 Cluster 302 cells stably expressing the red fluorescent protein, were seeded on the top surface of an organotypic brain slice culture of mouse brain, as described in the Materials and Methods section. Block 1 and 2 represent two independent organotypic brain slice cultures. The right panel shows phase contrast picture of red fluorescent TG1 Ctrl and TG1 Cluster 302 cells seeded on the top surface of the MBS (block 2). After 1 month, each organotypic culture was embedded in paraffin and sliced. Cell infiltration and growth within the neural host tissue was visualized by tracking the red fluorescent protein. Dashed lines mark the neural tissue boundaries. The histogram in the middle panel represents a measurement of the fluorescence intensity quantified using the Image $\mathrm{J}$ software (NIH, http://rsb.info.nih.gov/ii)). The values were compared with the background fluorescence measured in the same conditions in unseeded MBSs 
a

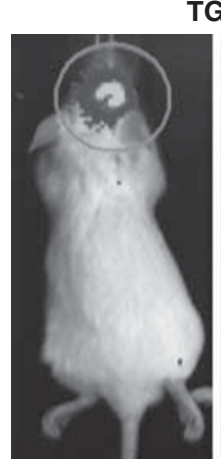

G1 Ctrl- mouse \#1

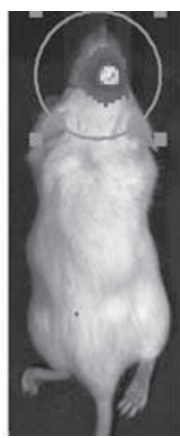

TG1 Ctrl- mouse \#2

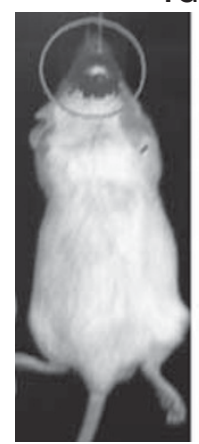

Day 14

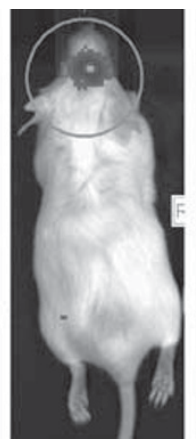

Day 21
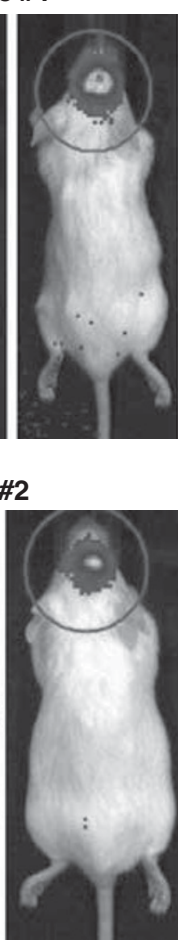

Day 30

Number of days following xenograft

TG1 Cluster 302- mouse \#1

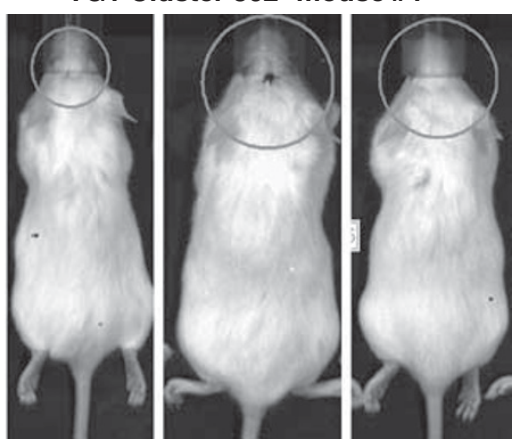

TG1 Cluster 302- mouse \#2

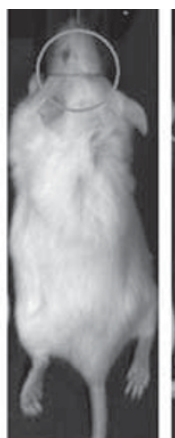

Day 14

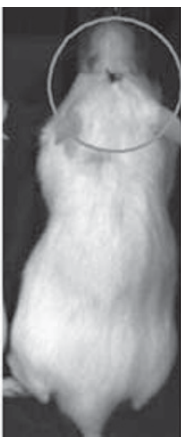

Day 21

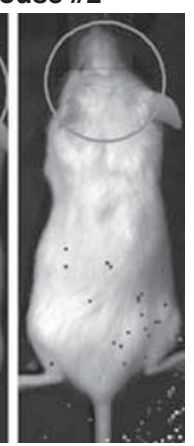

Day 30

Number of days

following xenograft

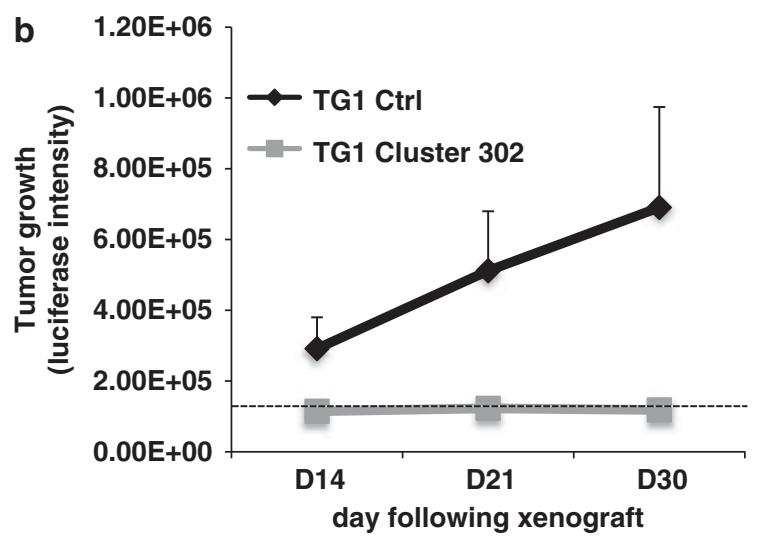

Figure 5 The miR-302-367 cluster inhibits tumor development in vivo. TG1 Ctrl and TG1 Cluster 302 cells were orthotopically implanted into the striatum of male NOD.CB17-Prkdcscid/NCrHsd mice. Tumors were visualized (a) and their growth was quantified (b) as described in the Materials and Methods section

(Figure 6c). Similar results were obtained using TG6 Ctrl and TG6 Cluster 302\#1 cells (Supplementary Figures 4A and B). To assess whether the miR-302 directly mediates CXCR4 expression, we cloned the $3^{\prime} U T R$ region of CXCR4 mRNA and fused it to a luciferase reporter gene; this construct was then transfected in 293T cells along with synthetic miR-302a or miR-23 (used as control non-relevant miR). Our results showed a strong repression of luciferase activity in the case of miR-302a (Figure 6d). This repression did not occur in the presence of miR-23 or when using a construct corresponding to the CXCR4 3'UTR lacking the miR-302a binding site. Therefore, these results argued for a direct role of miR-302 in the regulation of $\mathrm{CXCR} 4$ expression. Migration assays performed using Boyden chambers (Corning Incorporated, Corning, NY, USA) further confirmed CXCR4 inhibition in TG Cluster 302 cells, as no migration was observed in these cell lines (Figure 6f and Supplementary Figure 4C). Interestingly, cell migration resumed when CXCR4 expression was restored by stable expression of a CXCR4 construct lacking the $3^{\prime} U T R$ region (Supplementary Figure 4D). These results demonstrate that the miR-302-367 cluster promotes strong functional inhibition of the CXCR4 pathway.

CXCR4 constitutes one of the major miR-302-367 cluster targets involved in the suppression of GiCs properties. To assess the effects of CXCR4 inhibition in 
a

\begin{tabular}{|l|l|l|l|l|}
\hline TARGETS & miR-302a & miR-302b & miR-302c & miR-302d \\
\hline Seed positiion in CXCR4 3'UTR & $392-398$ & $392-398$ & $392-398$ & $392-398$ \\
\hline Seed positiion in SDF1 3'UTR & $159-167$ & $\begin{array}{l}159-167 \\
363-369\end{array}$ & $\begin{array}{l}159-167 \\
290-296\end{array}$ & $159-167$ \\
\hline
\end{tabular}
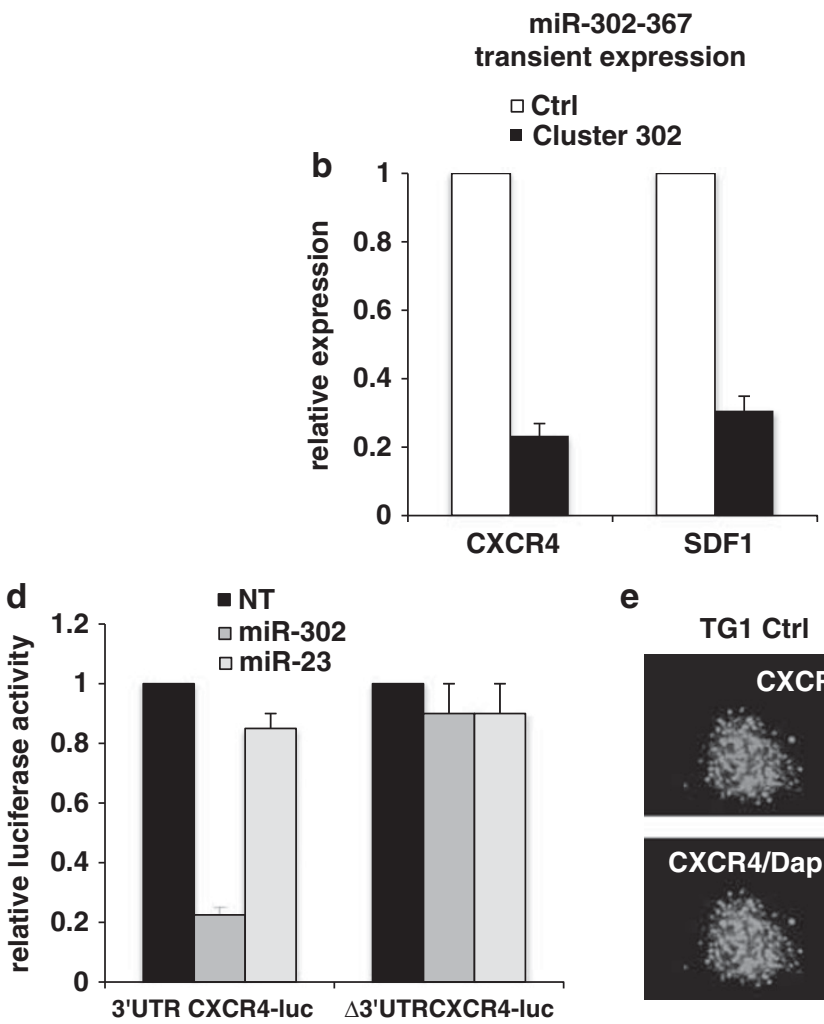

e

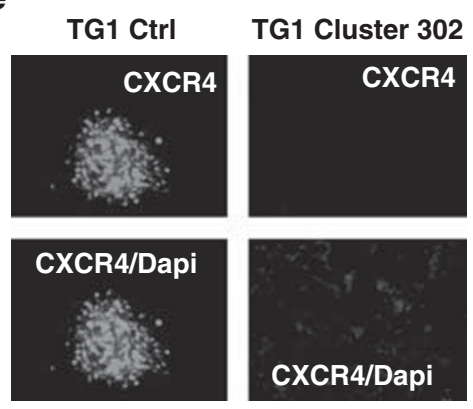

miR-302-367

stable expression

$\square$ Ctrl + siLUC

- Cluster $302+$ siLUC

C $\square$ Cluster $302+$ clear $\mathrm{miR}-302 \mathrm{a} / \mathrm{b}$

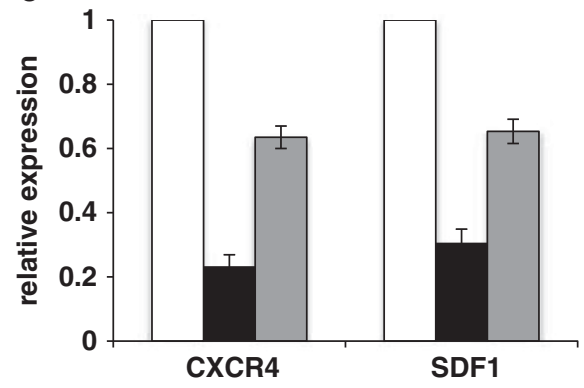

$\mathbf{f}$

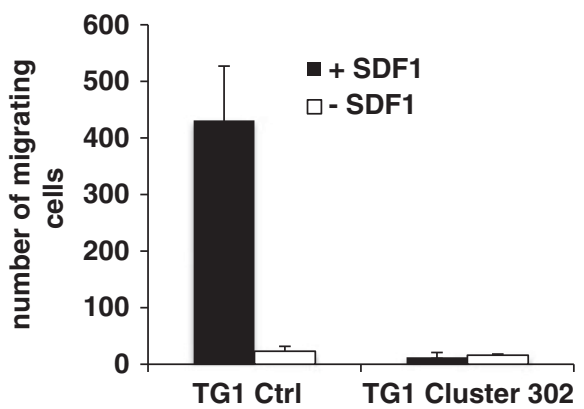

Figure 6 The miR-302-367 cluster promotes CXCR4 functional inhibition. (a) In silico analysis revealing the position of putative miR-302-367 cluster interactions on CXCR4 and SDF1 3'UTR. (b) TG1 cells were transiently transfected either with the miR-302-367 cluster construct (Cluster 302) or with a non-relevant small non-coding RNA as control (Ctrl).After 4 days transfection, total RNA was extracted and the level of expression of CXCR4 and SDF1 mRNA was assessed by QPCR. Results are represented as relative expression compared with the Ctrl. (c) TG1 Cluster 302 cells were transfected either with a non-relevant siRNA siLUC or with a pool of anti-miR specific for miR-302a and 302b (clear miR-302a/b). Total RNA was extracted and the level of expression of CXCR4 was assessed by QPCR. The results are expressed as relative expression compared with siLUC-transfected TG1 Ctrl cells. Error bars are derived from three independent experiments. (d) 293T cells were transiently transfected with a construct expressing the luciferase gene fused to the WT CXCR4 3'UTR or lacking the miR-302 binding site along with miR-302a or the non-relevant miR-23. (e) CXCR4 protein expression was assessed by immunofluorescence in both TG1 Ctrl and TG1 Cluster 302 cells. Nuclei were stained with DAPI. (f) Boyden chambers assays were performed using TG1 Ctrl and TG1 Cluster 302 cells in the presence or absence of the CXCR4 ligand SDF1

TG1 cells, we used the non-peptide antagonist AMD3100 or an anti-CXCR4-blocking antibody to prevent the binding of ligands. Treated and untreated TG1 cells were seeded on the surface of MBSs to evaluate their infiltrative properties. Although control untreated cells strongly and deeply interacted with the brain tissue, both TG1 Cluster 302 and TG1 cells deficient of functional CXCR4 did not, and remained as non-adherent spheres (Figure 7). Importantly, analysis of paraffin sections revealed that only the control TG1 cells were able to infiltrate and migrate into the cerebral tissue (Figure 7). The fact that similar results were obtained using TG1 cluster 302 cells or TG1 cells deficient of functional CXCR4 demonstrated that the miR-302-367 cluster-mediated CXCR4 inhibition was the main regulatory mechanism required to suppress GiCs infiltrative properties. As SDF1/CXCR4 has been described as a promoter of glioma cells proliferation, ${ }^{17}$ we reasoned that the reduced clonal proliferation of TG Cluster 302 cells (Figure 4a and Supplementary Figure 3D) might be the result of CXCR4 inhibition. Therefore, we performed clonal proliferation assays, using TG1 cells cultured either in their proliferation medium or in presence of anti-CXCR4-blocking antibody. Our results showed that inhibition of the CXCR4 pathway led to a drastic loss of clonal efficiency (Figure 8a). We then investigated the molecular mechanism behind the reduction of clonal proliferation induced by CXCR4 impairment. As 


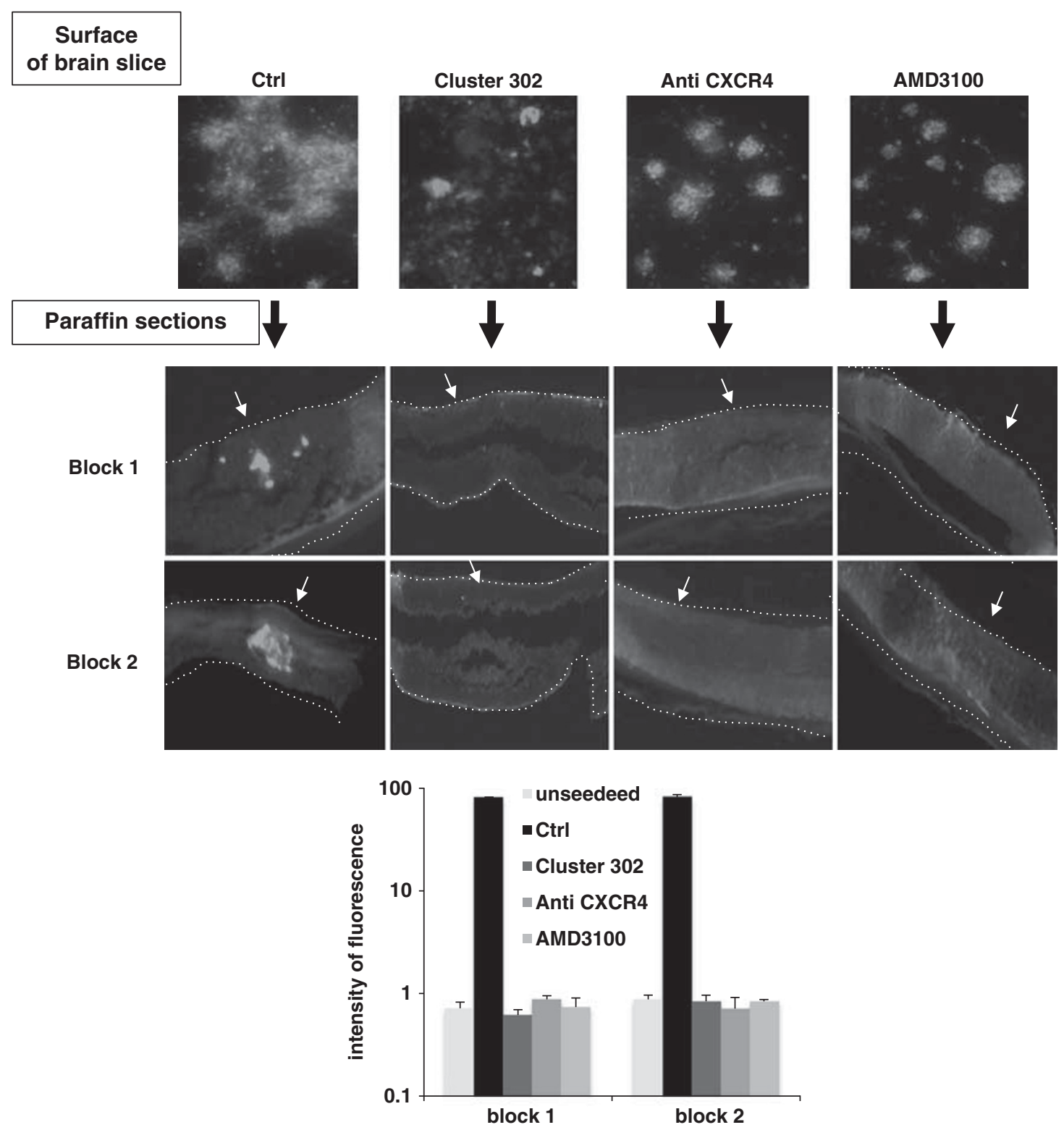

Figure 7 CXCR4 functional inhibition suppresses TG1 infiltration within a cerebral tissue. TG1 Ctrl cells were pre-incubated or not with the CXCR4 inhibitor AMD3100 or with anti-CXCR4-blocking antibody for $24 \mathrm{~h}$. These cells, as well as TG1 Cluster 302 cells (Cluster 302), were then seeded on the surface of a MBS. The upper panels (surface of brain slice) show cell interactions with the surface of the brain slice. The lower panels (paraffin sections) represent paraffin sections showing cells infiltration only in the control condition (Ctrl). Dashed lines mark the neural tissue boundaries. Block1 and 2 show the section of two different MBSs. The white arrows indicate the surface on which the cells were seeded. The histogram represents a measurement of the fluorescence intensity quantified using the Image $\mathrm{J}$ software. The values were compared with the background fluorescence measured in the same conditions in unseeded MBSs

previous reports demonstrated that the SHH-GLI1-NANOG network is crucial for glioma cancer stem cells self-renewal and tumorigenic properties, ${ }^{13,15,18,19}$ we assessed the expression of this gene network in TG1 cells stably expressing the miR-302-367 cluster or grown in the presence of anti-CXCR4-blocking antibody or AMD3100. QPCR analysis revealed that stable expression of the miR302-367 cluster or functional inhibition of CXCR4 caused reduction of both $\mathrm{SHH}$ and GLI1 expression (Figure $8 \mathrm{~b}$ ). Consistently, while SHH and NANOG were clearly detected in control cells by immunofluorescence, they were almost undetectable in cells with impaired CXCR4 function and in cells stably expressing the miR-302-367 cluster (Figure 8c). Consistent with the drastic loss of clonal proliferation (Figure 8e), PBK/TOPK kinase and its substrate (the histone H3 ser10), which are expressed and phosphorylated in dividing cells, were also suppressed (Figure 8f). The restoration of CXCR4 expression by stable overexpression of a CXCR4 construct lacking the $3^{\prime} \mathrm{UTR}$ region, rescued both $\mathrm{SHH}$ and NANOG protein expression, the Hedgehog pathway, $50 \%$ of PBK/TOPK, and histone H3 ser10 expression, and therefore a part of TG1 Cluster 302 


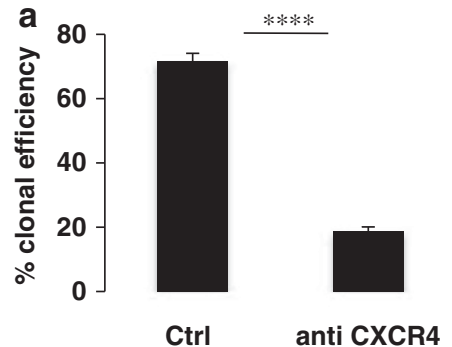

C

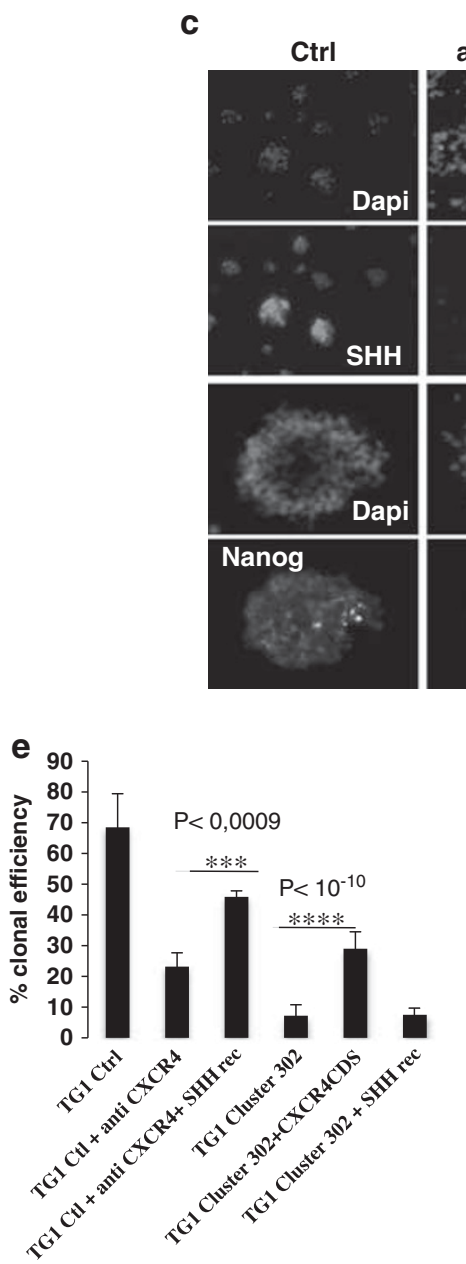

e 90

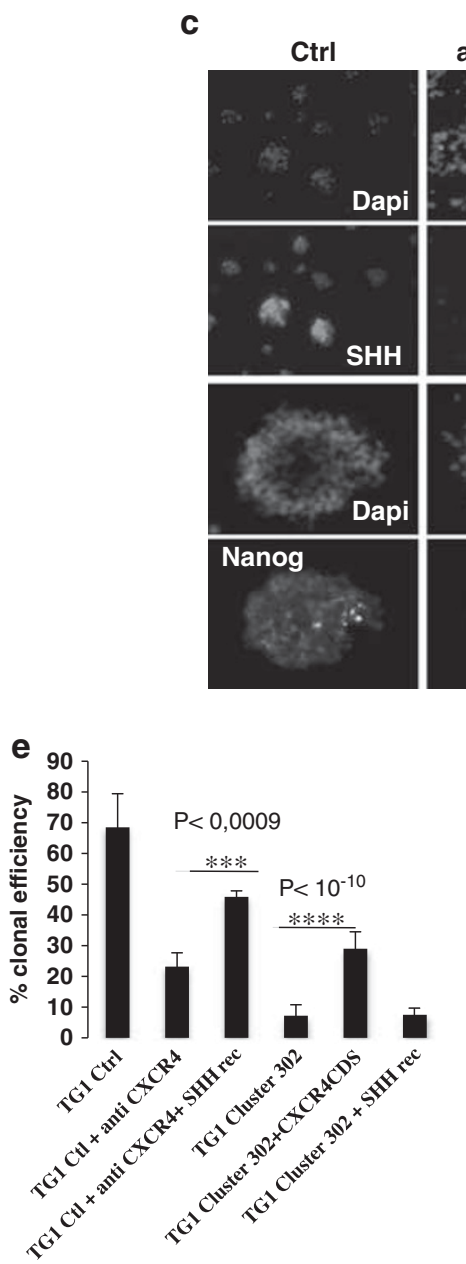

anti CXCR4
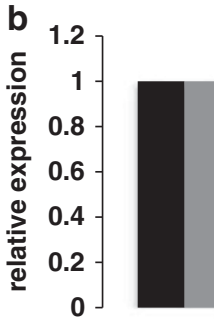

Ctrl
๑ SHH

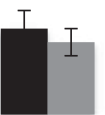

AMD3100
GLI1
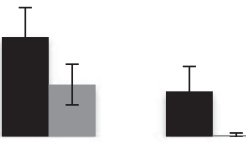

anti CXCR4 TG1 cluster TG1 cluster 302

$302+$ CXCR4CDS

Cluster 302

d

f

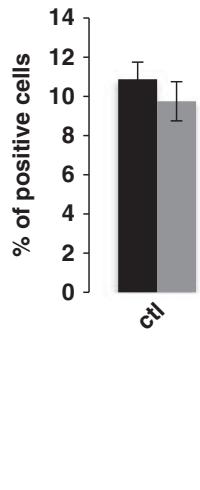

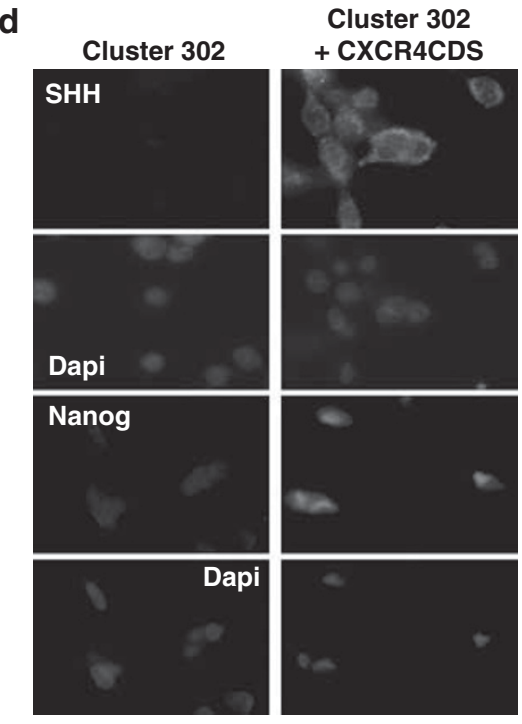

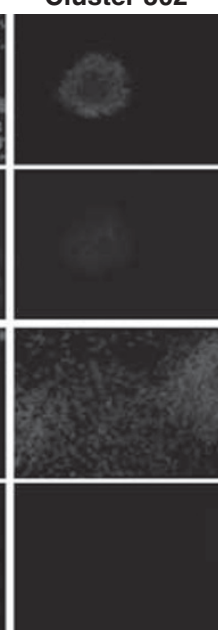

g

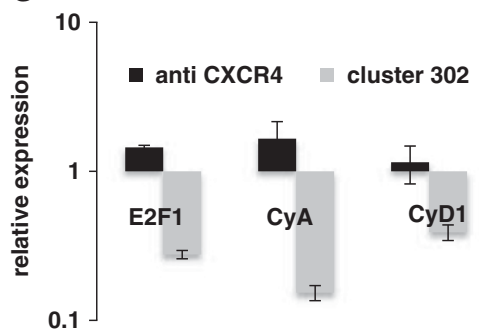

Figure 8 CXCR4 functional inhibition impairs the SHH-GLI1-NANOG network leading to a drastic loss of clonal proliferation. (a) TG1 cells were treated with anti-CXCR4blocking antibody (anti CXCR4) or not (Ctrl). A clonogenical assay was then performed by seeding 10 cells/well, as described in the Materials and Methods section. In all, 10 neurospheres/well corresponded to a clonal efficiency of $100 \%$. Error bars are derived from three independent experiments. Statistical analysis was performed using Student's $t$-test; ${ }^{* * * *}$ corresponds to a $P$-value of $3.4 \times 10^{-13}$ and indicates a highly signicant difference between the control and treated cells. (b) SHH and GLI1 mRNA expression levels were assessed by QPCR using Taqman probes in cells treated with AMD3100 or anti-CXCR4 antibody, in control cells (Ctr), and in TG1 cluster 302 cells in which CXCR4 expression was restored by a CXCR4 construct lacking the $3^{\prime}$ UTR sequences. (c) SHH and NANOG expression at the protein level was assessed by immunofluorescence in TG1 cells treated with anti-CXCR4 or not (Ctr) and in TG1 Cluster 302 cells. (d) SHH and NANOG expression at the protein level was assessed in TG1 Cluster 302 cells in which CXCR4 expression was restored by a CXCR4 construct lacking the $3^{\prime}$ UTR sequences. (e) TG1 cells treated with anti-CXCR4-blocking antibody (anti CXCR4) or not (Ctrl), and TG1 cluster 302 cells were grown in the presence or absence of recombinant SHH protein. A clonogenical assay was performed by seeding 10 cells/ well as described in the Materials and Methods section. In all, 10 neurospheres/well corresponded to a clonal efficiency of $100 \%$. Error bars are derived from three independent experiments. Statistical analysis was performed using Student $t$-test; ${ }^{* * *}$ corresponds to a $P$-value of $10^{-4}$ and indicates a highly signicant difference between the control and treated cells. (f) Quantification of TG1 cells treated with anti-CXCR4 or not (Ctrl) and TG1 Cluster 302 cells positive for PBK/TOPK kinase or Histone H3Ser10. (g) E2F1, CyA, and CyD1 mRNA expression were assessed in TG1 cells treated or not with anti-CXCR4 and in TG1 Cluster 302 cells. Results are expressed as relative expression compared with untreated TG1 cells and TG1 Ctrl cells stably expressing a non-relevant small non-coding RNA, respectively 
cells clonal proliferation (Figures $8 b, d$, e, and f). These results revealed that a functional CXCR4 pathway is necessary to maintain the SHH-GLI1-NANOG network and self-renewal properties. In addition, they revealed the strong ability of the miR-302-367 cluster to disrupt the SHH-GLI1NANOG network, in part through CXCR4 inhibition. We then restored $\mathrm{SHH}$ expression in TG1 cells either impaired for CXCR4 function or stably expressing the miR-302-367 cluster using an exogenous recombinant protein. Our results showed that exogenous recombinant $\mathrm{SHH}$ was able to increase clonal proliferation of cells lacking functional CXCR4 (Figure 8e). However, exogenous recombinant $\mathrm{SHH}$ failed to rescue clonal proliferation of TG1 cells stably expressing the miR-302-367 cluster (Figure 8e). Exogenous recombinant $\mathrm{SHH}$ was therefore sufficient to bypass CXCR4 inhibition, confirming the importance of this pathway in the control of self-renewal. The fact that recombinant $\mathrm{SHH}$ had no effect on cells stably expressing the miR-302-367 cluster suggests a strong ability of these miRs to inhibit multiple pathways involved in cell proliferation. A recent report and in silico analyses have identified various proteins involved in the regulation of cell-cycle progression - E2F1, cyclin A (CyA) and cyclin D1 (CyD1) - as direct targets of the miR-302-367 cluster. ${ }^{20}$ Therefore, we assessed the expression of such proteins in TG1 lacking functional CXCR4 or stably expressing the miR-302-367 cluster. QPCR revealed that expression of the miR-302-367 cluster caused a drastic downregulation of E2F1, CyA, and CyD1 mRNA levels, whereas CXCR4 inhibition did not (Figure 8g). These results demonstrate that expression of the miR-302-367 cluster compromises both cell infiltration and self-renewal properties by inducing a cascade of inhibitory events leading to CXCR4 repression and consequent disruption of the SHH-GLI1NANOG network. In addition, they demonstrate that the miR-302-367 cluster is able to directly interfere with the expression of proteins of the cell-cycle machinery.

\section{Discussion}

GiCs represent an attractive target for the treatment of highgrade brain tumors such as GBM. A possible approach to target GiCs consists in forcing them into a more differentiated phenotype characteristic of the tumor bulk. ${ }^{10}$ We demonstrated the ability of the miR-302-367 cluster to compromise the maintenance of $\mathrm{GiCs}$ and to reduce their infiltration within a cerebral tissue. Stable expression of the miR-302-367 cluster was sufficient to strongly inhibit the clonogenicity of GiCs and to promote loss of stem-like proteins including Oct4 and Nanog, ${ }^{13,21}$ as well as Sox1 and $\mathrm{SHH}$ - known to be involved in adult normal neural stem cell and cancer stem cells self-renewal and maintenance. ${ }^{15,22-27}$ Consistent with our results, a previous report showed the importance of the miR302-367 in cell differentiation by describing its role in the promotion of mesendodermal fate specification. ${ }^{28}$ On the other hand, other reports described the miR-302-367 cluster as a stemness determinant in human embryonic stem cells $(E S C)^{29}$ and in inducible pluripotent stem cells (IPS) derived from human skin cancer cells. ${ }^{30}$ In this context, the miR-302367 promoter was transcriptionally regulated by the stemness transcription factors Oct4, Sox2, and Nanog; therefore, its expression in the ESC compartment and during early stages of mouse development was restricted. ${ }^{20,31}$ In GiCs, the expression of the miR-302-367 cluster occurs concomitantly with the suppression of Oct4 and Nanog, as well as with the loss of self-renewal. This suggests the existence of a mechanism distinct from that observed in pluripotent cells and particularly efficient in maintaining GiCs stem-like properties through the repression of these miRNAs. In addition, the drastic loss of the stem-like signature mediated by miR-302367 cluster expression suggests drastic epigenetic changes, indicating that these miRNAs might exhibit powerful regulatory potential.

Our ex-vivo and in vivo models demonstrated that the miR-302-367 cluster is able to suppress GiCs infiltration and tumorigenicity. This is the most important aspect of the miR-302-367 cluster activity, as tumor glial cell infiltration represents one of the main causes of the short survival time observed in GBM. In this context, we showed that the miR-302-367 cluster affects migration and clonal proliferation through a drastic inhibition of the chemokine receptor CXCR4 and its ligand SDF1. The binding of SDF1 to CXCR4 is known to trigger divergent signaling pathways such as PLC, PI3K/ $A K T$, and MAPK, resulting in a variety of physiological responses including gene transcription, motility, survival, and proliferation. ${ }^{32}$ Numerous studies reported that CXCR4 has a role in proliferation and motility, thereby contributing to the development of highly malignant human gliomas and to tumor aggressiveness. In addition, CXCR4 expression strongly correlates with poor survival. ${ }^{16,17,33}$ In GiCs, the network composed of SHH-GLI-NANOG proteins represents another important pathway involved in the regulation of proliferation, self-renewal, and expression of stemness genes. ${ }^{13,15,18,19} \mathrm{SHH}-\mathrm{GLI}$ signaling acts through positive regulators of proliferation such as the PBK/TOPK protein a PDZ-binding kinase involved in mitosis, ${ }^{34}$ known to promote growth through the phosphorylation of histone $\mathrm{H} 3$ at Ser10. ${ }^{35,36}$ In this study, we demonstrated that impairment of CXCR4 function leads to the drastic repression of hedgehog signaling, therefore compromising NANOG, PBK/TOPK expression and histone $\mathrm{H} 3$ phosphorylation at Ser10, contributing to loss of clonal proliferation. The significant rescue of clonal proliferation mediated by exogenous recombinant $\mathrm{SHH}$ protein, confirms the involvement of the CXCR4 pathway in the positive regulation of the SHH-GLI1-NANOG network, and its contribution in self-renewal. These results emphasize the potential of CXCR4 functional inhibition as a therapeutic approach for GBM. Indeed, CXCR4 inhibition would prevent tumor regrowth by inhibiting vasculogenesis ${ }^{37}$ while affecting GiCs stem-like and tumorigenic properties. In a context where novel strategies for GBM treatment are much needed, the miR-302-367 cluster appears therefore to be a promising therapeutic target, as it downregulates the CXCR4 pathway. This is sufficient to trigger a cascade of inhibitory events that leads to the disruption of the SHH-GLI1-NANOG network, the blockade of cell infiltration, and suppression of GiCs self-renewal and stem-like signature. Our data demonstrate that the miR-302-367 cluster is not only able to inhibit the CXCR4 pathway, but it may also directly repress the cell-cycle machinery by strongly inhibiting positive regulators such as E2F1, CyA, and CyD1, which were already described 
as its putative targets. ${ }^{20}$ Thus, the miR-302-367 cluster not only affects GiCs stem-like and infiltrative properties, but also specifically inhibits the cell-cycle machinery. The capacity of affecting different pathways involved in the promotion of cell proliferation diminishes the likelihood of cell adaptation and compensatory mechanisms, and therefore makes the miR-302-367 cluster an even more attractive therapeutic target.

\section{Materials and Methods}

Reagents and antibodies. Cell culture reagents, pENTR cloning kit (catalog number K2400-20), LR Clonase II (catalog number 11791-020), Superscript II reverse transcriptase (catalog number 18064-014), and Trizol were purchased from Invitrogen (Cergy Pontolse, France). Foetal calf serum was from Hyclone (Brebières, France). Hoechst 33342, U0126, TWS119, and anti-B3Tubulin antibody (catalog number CBL412-diluted $1 / 100^{\circ}$ ) were purchased from Sigma (Lyon, France). Anti-SHH (catalog number sc1194-diluted $1 / 50^{\circ}$ ) and anti-Oct4 (catalog number sc9081-diluted $1 / 50^{\circ}$ ) antibodies were purchased from Santa-Cruz Biotechnology (Heidelberg, Germany). Anti-Nanog (catalog number AF1997$5 \mu \mathrm{g} / \mathrm{ml}$ ), anti-CXCR4-blocking antibody (catalog number MAB170-diluted $1 / 100^{\circ}$ ), anti-CXCR4 (catalog number MAB 173), and anti-Olig2 (catalog number AF2418, $10 \mu \mathrm{g} / \mathrm{ml}$ ) antibodies were from R\&D Systems. The CXCR4 chemical inhibitor AMD3100 (catalog number A5602) was purchased from Sigma-Aldrich (Lyon, France). Anti-GFAP (catalog number $2203 \mathrm{PGF}-1 / 200^{\circ}$ ) antibody was purchased from AbCys (Paris, France). Anti-CA (NCL-cyclin A-diluted $1 / 250^{\circ}$ ) was from Novocastra (Nanterre, France). Anti-NeuN (MAB377-diluted $1 / 150^{\circ}$ ) was from Chemicon (Chemicon International, Temecula, CA, USA). Anti-PKB (ab59327diluted $1 / 100^{\circ}$ ) and anti-histone H3Ser10 (catalog number Ab 5176) were purchased from Abcam. Anti-species secondary antibodies coupled to Alexa 488 (diluted $1 / 500^{\circ}$ ) or Alexa 546 (diluted $1 / 500^{\circ}$ ) were purchased from Alexa (Dusseldorf, Germany). Gel/mount was purchased from Biomedia (Roskilde, Denmark). Taqman Reverse transcription microRNA kit (catalog number 4366596), Universal Taqman PCR Master Mix, Taqman low-density array (TLDA array), and Taqman probes were purchased from Applied Biosystems (Villebon sur Yvette, France). Enhanced chemiluminescence detection reagent was from BioRad (Marnes la Coquette, France).

Cell culture. The GiCs primary cell lines TG1, TG6, GB1, and \#1056 were isolated from human glioblastoma. ${ }^{14}$ When kept as self-renewing GiCs, neurospheres were grown in NS34+ medium containing EGF and bFGF (DMEM-F12 1/1, glutamine $10 \mathrm{~mm}$, Hepes $10 \mathrm{~mm}$, Sodium bicarbonate $0.025 \%$, N2, G5, and B27). The medium for cell differentiation (MFCS) was composed of DMEMF12, glutamine $10 \mathrm{mM}$, Hepes $10 \mathrm{mM}$, Sodium bicarbonate $0.025 \%$, and FCS $0.5 \%$. In differentiation experiments, the neurospheres were dissociated and 500000 single cells were cultured in MFCS. When necessary, the cells were incubated in NS34-minimum medium depleted of EGF and bFGF (DMEM-F12 1/1, glutamine $10 \mathrm{mM}$, Hepes $10 \mathrm{mM}$, Sodium bicarbonate $0.025 \%$ ). When indicated, the cells were treated with anti-CXCR4 antibody $(1-10 \mu \mathrm{g} / \mathrm{ml})$ or AMD3100 $(10-100 \mathrm{nM})$.

Plasmid constructs and stable cell lines. The miR-302-367 cluster was amplified from genomic DNA by PCR (Forward primer: $5^{\prime}$-GGCTGAAGTCCCTG CCTTTTACCC-3'; Reverse primer: 5'-TGGCTTAACAATCCATCACCATTGC- ${ }^{\prime}$ ) and cloned into a pENTR commercial vector (Invitrogen, Life Technology, Cergy Pontoise, France). Subcloning in the 2K7 blasticidin lentiviral vector (2K7BSD) was realized by recombination in the presence of $L R$ clonase II. Lentiviral particles were produced transfecting the 293T cell line with the 2K7BSD-Cluster mir302 or 2K7BSD-shLuc constructs along with the packaging vectors. After lentiviral infection, cell lines stably expressing the miR-302-367 cluster (TG1cluster 302 and TG6 cluster 302\#1) or the control shLuc (TG1 Ctrl and TG6 Ctrl) were selected in appropriate blasticidin NBE medium $(1 \mu \mathrm{g} / \mathrm{mL})$ for at least 15 days. Two stable cell lines were developed from independent viral productions/infections and exhibited identical behaviors. Cells stably expressing the Red Fluorescent Protein (RFP) were obtained after 2K7BSD-RFP lentiviral particles infection. To restore CXCR4 expression, TG1 Cluster 302 and TG6 Cluster 302\#1 cells were transduced with a commercially available CXCR4 lentivirus coding for a construct lacking the $3^{\prime} U T R$ of the gene.
Immunofluorescence. Cells were grown on polylysine-coated glass slides in NBE, MFCS, or TG1-cluster-conditioned medium. At indicated time points, cells were washed twice with ice-cold PBS, fixed with methanol $5 \mathrm{~min}$ at $-20^{\circ} \mathrm{C}$, and washed again. Blocking and hybridization were performed in PBS containing $10 \%$ FCS and $0.1 \%$ Triton X100 with the following primary antibodies: anti-SHH at $1 / 50^{\circ}$ (N19), antiOct4 diluted $1 / 50^{\circ}(\mathrm{H}-134)$, anti-Nanog at $5 \mu \mathrm{g} / \mathrm{ml}$, anti-GFAP diluted $1 / 250^{\circ}$, antiOlig2 at $10 \mu \mathrm{g} / \mathrm{ml}$, anti-B3tubulin diluted $1 / 100^{\circ}$, anti-CyA $\left(1 / 250^{\circ}\right)$, anti-PBK (diluted $1 / 150^{\circ}$ ), anti-CXCR4 (diluted $1 / 100^{\circ}$ ), or anti-NeuN (diluted $1 / 100^{\circ}$ ). After $1 \mathrm{~h}$ of incubation at room temperature, cells were washed three times with PBS and stained for $30 \mathrm{~min}$ at room temperature with species-specific fluorophore-coupled antibodies. At the same time, nuclei were stained with Hoechst $33342(1 \mu \mathrm{g} / \mathrm{ml})$. The slides were washed twice with PBS, once with distilled water, and finally mounted with gel/mount. Immunofluorescence and transmission light pictures were taken with a Nikon eclipse Ti microscope (Nikon, Champigny sur Marne, France).

Clonogenical assay. Neurospheres were dissociated by gently pipeting up and down to obtain a single cell suspension. In all, 10 cells were seeded in each well of 96-wells plates in NBE/NS34 + medium containing, when indicated, antiCXCR4-blocking antibody. After 1 month, each well was examined and the number of neurospheres/well was counted. Experiments were repeated three independent times.

Quantitative real-time RT-PCR. RNA was extracted using Trizol reagent (Invitrogen, Invitrogen SARL BP 9695613). MicroRNA and mRNA expression levels were quantified by two step real-time RT-PCR. Reverse transcription steps were performed with Superscript II reverse transcriptase and Taqman Reverse transcription microRNA kit for mRNA and miRNA, respectively, following the manufacturer's instructions. Real time PCRs were performed using universal Taqman PCR Master Mix. U54 expression was used as internal control to determine the relative expression of each gene. Fold changes were estimated using the CT method. miRNA screening was performed using TAQMAN TLDA arrays (Applied Biosystems), following the manufacturer's instructions. The results have been normalized using expression of small nucleolar RNA, according to the manufacturer's protocol. The fold changes were calculated and normalized using the CT method.

Orthotopic xenografts. TG1 Ctrl and TG1 Cluster 302 cells $\left(2 \times 10^{5}\right)$ were resuspended in $5 \mu$ of Hanks balanced salt solution (Invitrogen) and stereotactically implanted unilaterally into the striatum of male NOD.CB17-Prkdcscid/NCrHsd mice (Harlan, rms.fr@ harlan.com). The cells stably expressed a luciferase reporter gene, which allowed tumor detection in living animals. Two groups of five mice were injected. Cell survival and tumor growth were monitored and quantified in the living animals up to 30 days using the IVIS Lumina II system (Caliper Life Sciences, Hopkinton, MA, USA).

Organotypic MBS culture. Brains were dissected from new-born mice, embedded in $4 \%$ agar-agar artificial cerebroSpinal fluid $(\mathrm{NaCl} 124 \mathrm{mM}, \mathrm{KCl} 3 \mathrm{mM}$, $\mathrm{NaHCO}_{3} 26 \mathrm{mM}, \mathrm{CaCl}_{2} 2 \mathrm{mM}, \mathrm{MgSO}_{4} 1 \mathrm{mM}, \mathrm{KH}_{2} \mathrm{PO}_{4} 1.25 \mathrm{mM}$, glucose $10 \mathrm{mM}$ ), and cut in slices of $400 \mathrm{~mm}$ thickness using a vibratome. The slices were placed on a Millicell-CM $(0.4 \mu \mathrm{m})$ culture plate, inserted and maintained under air-liquid interface conditions for more than 3 weeks without any necrosis.

Paraffin embedding. Organotypic cultures (brain slides) or tissues were fixed with $4 \%$ paraformaldehyde for 20 min at room temperature, then washed with PBS. Samples were subsequently dehydrated with the following sequence of incubations: ethanol $70 \% 15 \mathrm{~min}$, twice; ethanol $90 \% 15 \mathrm{~min}$; ethanol $95 \% 15 \mathrm{~min}$; ethanol $100 \%$ $5 \mathrm{~min}$, thrice; Roticlear $5 \mathrm{~min}$.

Ex vivo tumorigenesis assay. About 10 neurospheres from TG1 Ctrl and TG1 Cluster 302 cells stably expressing the red fluorescent protein were seeded at the top surface of the MBS and cultured under air-liquid interface conditions for 3 weeks. Cell infiltration and growth was visualized by tracking the red fluorescent protein signal using a Nikon eclipse Ti microscope. When indicated, a drop of medium containing anti-CXCR4-blocking antibody or the chemical inhibitor AMD3100 was added to the brain slices every 4 days.

\section{Conflict of Interest}

The authors declare no conflict of interest. 
Acknowledgements. This work was supported by grants from Association pour la Recherche sur le Cancer (subvention 3161), Association Sauvons Laura, Agence Nationale pour la Recherche (ANR jeune chercheur), INSERM, UNSA OSEO/VALORPACA. We thank Dr. Rassoulzadegan's lab (Inserm U636, F-06108 Nice, France) for their contribution in our organotypic culture model of mouse brain.

1. Stupp R, Mason WP, van den Bent MJ, Weller M, Fisher B, Taphoorn MJ et al. Radiotherapy plus concomitant and adjuvant temozolomide for glioblastoma. N Engl J Med 2005; 352: 987-996.

2. Wen PY, Kesari S. Malignant gliomas in adults. N Engl J Med 2008; 359: 492-507.

3. Shannon RP, Sayre JW, Sayre JJ. Patterns of care for adults with malignant glioma. Jama 2005; 293: 2469-2470; author reply 2470.

4. Hegi ME, Diserens AC, Godard S, Dietrich PY, Regli L, Ostermann S et al. Clinical tria substantiates the predictive value of 0-6-methylguanine-DNA methyltransferase promote methylation in glioblastoma patients treated with temozolomide. Clin Cancer Res 2004; 10 : 1871-1874.

5. Germano I, Swiss V, Casaccia P. Primary brain tumors, neural stem cell, and brain tumor cancer cells: where is the link? Neuropharmacology 2010; 58: 903-910.

6. Passegue $\mathrm{E}$, Jamieson $\mathrm{CH}$, Ailles LE, Weissman IL. Normal and leukemic hematopoiesis: are leukemias a stem cell disorder or a reacquisition of stem cell characteristics? Proc Nat Acad Sci USA 2003; 100(Suppl 1): 11842-11849.

7. Smalley M, Ashworth A. Stem cells and breast cancer: a field in transit. Nat Rev Cancer 2003; 3: 832-844.

8. Wu W 2010 Patents related to cancer stem cell research. Recent Pat DNA Gene Seq 4: 40-45

9. Visvader JE, Lindeman GJ. Cancer stem cells in solid tumours: accumulating evidence and unresolved questions. Nat Rev Cancer 2008; 8: 755-768.

10. Piccirillo SG, Vescovi AL. Bone morphogenetic proteins regulate tumorigenicity in human glioblastoma stem cells. Ernst Schering Found Symp Proc 2006; 5: 59-81.

11. Rao M. Conserved and divergent paths that regulate self-renewal in mouse and human embryonic stem cells. Dev Biol 2004; 275: 269-286.

12. Wang $Y$, Medvid R, Melton C, Jaenisch R, Blelloch R. DGCR8 is essential for microRNA biogenesis and silencing of embryonic stem cell self-renewal. Nat Genet 2007; 39 380-385

13. Zbinden M, Duquet A, Lorente-Trigos A, Ngwabyt SN, Borges I, Ruiz IAA. NANOG regulates glioma stem cells and is essential in vivo acting in a cross-functional network with GLI1 and p53. Embo J 2010; 29: 2659-2674.

14. Patru C, Romao L, Varlet P, Coulombel L, Raponi E, Cadusseau J et al. CD133, CD15/ SSEA-1, CD34 or side populations do not resume tumor-initiating properties of long-term cultured cancer stem cells from human malignant glio-neuronal tumors. BMC Cancer 2010; 10: 66

15. Clement V, Sanchez P, de Tribolet N, Radovanovic I, Ruiz i Altaba A. HEDGEHOG-GLI signaling regulates human glioma growth, cancer stem cell self-renewal, and tumorigenicity. Curr Biol 2007; 17: 165-172.

16. Ehtesham M, Stevenson CB, Thompson RC. Preferential expression of chemokine receptor CXCR4 by highly malignant human gliomas and its association with poor patient survival. Neurosurgery 2008; 63: E820; author reply E820.

17. do Carmo A, Patricio I, Cruz MT, Carvalheiro H, Oliveira CR, Lopes MC. CXCL12/CXCR4 promotes motility and proliferation of glioma cells. Cancer Biol Ther 2010; 9: 56-65.

18. Shi Y, Sun G, Zhao C, Stewart R. Neural stem cell self-renewal. Crit Rev Oncol Hematol 2008; 65: 43-53.
19. Lai K, Kaspar BK, Gage FH, Schaffer DV. Sonic hedgehog regulates adult neural progenitor proliferation in vitro and in vivo. Nat Neurosci 2003; 6: 21-27.

20. Card DA, Hebbar PB, Li L, Trotter KW, Komatsu Y, Mishina Y et al. Oct4/Sox2-regulated miR-302 targets cyclin D1 in human embryonic stem cells. Mol Cell Biol 2008; 28: 6426-6438.

21. Ben-Porath I, Thomson MW, Carey VJ, Ge R, Bell GW, Regev A et al. An embryonic stem cell-like gene expression signature in poorly differentiated aggressive human tumors. Nat Genet 2008; 40: 499-507.

22. Alcock J, Sottile V. Dynamic distribution and stem cell characteristics of Sox1-expressing cells in the cerebellar cortex. Cell Res 2009; 19: 1324-1333.

23. Wegner M, Stolt CC. From stem cells to neurons and glia: a Soxist's view of neural development. Trends Neurosci 2005; 28: 583-588.

24. Palma V, Lim DA, Dahmane N, Sanchez P, Brionne TC, Herzberg CD et al. Sonic hedgehog controls stem cell behavior in the postnatal and adult brain. Development 2005 132: 335-344.

25. Elkouris M, Balaskas N, Poulou M, Politis PK, Panayiotou E, Malas S. et al. 2011 Sox maintains the undifferentiated state of cortical neural progenitor cells via the suppression of Prox1-mediated cell cycle exit and neurogenesis. Stem Cells 29: 89-98.

26. Salcido CD, Larochelle A, Taylor BJ, Dunbar E, Varticovski L. Molecular characterisation of side population cells with cancer stem cell-like characteristics in small-cell lung cancer. Br J Cancer 2010; 102: 1636-1644.

27. Wright MH, Calcagno AM, Salcido CD, Carlson MD, Ambudkar SV, Varticovski L. Brca1 breast tumors contain distinct CD44+/CD24- and CD133+ cells with cancer stem cell characteristics. Breast Cancer Res 2008; 10: R10.

28. Rosa A, Spagnoli FM, Brivanlou AH. The miR-430/427/302 family controls mesendodermal fate specification via species-specific target selection. Dev Cell 2009; 16: $517-527$.

29. Barroso-del Jesus A, Lucena-Aguilar G, Menendez P. The miR-302-367 cluster as a potential stemness regulator in ESCs. Cell Cycle 2009; 8: 394-398.

30. Lin SL, Chang DC, Chang-Lin S, Lin CH, Wu DT, Chen DT et al. Mir-302 reprograms human skin cancer cells into a pluripotent ES-cell-like state. Rna 2008; 14 2115-2124.

31. Barroso-delJesus A, Romero-Lopez C, Lucena-Aguilar G, Melen GJ, Sanchez L Ligero $G$ et al. Embryonic stem cell-specific miR302-367 cluster: human gene structure and functional characterization of its core promoter. Mol Cell Biol 2008; 28 6609-6619.

32. Teicher BA, Fricker SP. CXCL12 (SDF-1)/CXCR4 pathway in cancer. Clin Cancer Res 2010; 16: 2927-2931.

33. Ehtesham M, Mapara KY, Stevenson CB, Thompson RC. CXCR4 mediates the proliferation of glioblastoma progenitor cells. Cancer Lett 2009; 274: 305-312.

34. Dougherty JD, Garcia AD, Nakano I, Livingstone M, Norris B, Polakiewicz R et al. PBK/ TOPK, a proliferating neural progenitor-specific mitogen-activated protein kinase kinase. J Neurosci 2005; 25: 10773-10785.

35. Park JH, Lin ML, Nishidate T, Nakamura Y, Katagiri T. PDZ-binding kinase/T-LAK celloriginated protein kinase, a putative cancer/testis antigen with an oncogenic activity in breast cancer. Cancer Res 2006; 66: 9186-9195.

36. Hans F, Dimitrov S. Histone H3 phosphorylation and cell division. Oncogene 2001; 20 3021-3027.

37. Kioi M, Vogel H, Schultz G, Hoffman RM, Harsh GR, Brown JM. Inhibition of vasculogenesis, but not angiogenesis, prevents the recurrence of glioblastoma after irradiation in mice. J Clin Invest 2010; 120: 694-705. 